\title{
Études/Inuit/Studies
}

\section{La protection des noms de lieux et des sites sacrés autochtones, au niveau des instances internationales}

\section{Françoise Morin}

Volume 28, numéro 2, 2004

Espaces-Lieux-Noms

Spaces-Places-Names

URI : https://id.erudit.org/iderudit/013203ar

DOI : https://doi.org/10.7202/013203ar

Aller au sommaire du numéro

Éditeur(s)

Association Inuksiutiit Katimajiit Inc.

Centre interuniversitaire d'études et de recherches autochtones (CIÉRA)

ISSN

0701-1008 (imprimé)

1708-5268 (numérique)

Découvrir la revue

Citer ce document

Morin, F. (2004). La protection des noms de lieux et des sites sacrés autochtones, au niveau des instances internationales. Études/Inuit/Studies, 28(2), 203-209. https://doi.org/10.7202/013203ar d'utilisation que vous pouvez consulter en ligne. 


\section{Document}

\section{La protection des noms de lieux et des sites sacrés autochtones, au niveau des instances internationales}

Françoise Morin*

Les réflexions qui suivent ont été présentées lors du colloque «Autochtonie et Gouvernance» ${ }^{1}$, à la suite de la communication de Bernard Saladin d'Anglure sur «La toponymie religieuse et l'appropriation symbolique du territoire par les Inuit du Nunavik et du Nunavut». Elles visaient à resituer la démarche des Inuit dans le contexte plus global des revendications autochtones devant les instances internationales. Je les ai complétées par de nouvelles données recueillies à l'ONU en décembre 2004.

La toponymie religieuse fait en effet partie du patrimoine culturel que les Peuples Autochtones cherchent à préserver pour le transmettre aux générations futures. A cette fin, ils en font un élément essentiel de leurs revendications auprès des instances onusiennes. Depuis une vingtaine d'années, différents organismes du système des Nations Unies ont commencé à prendre en compte cette préoccupation autochtone.

Tout débute à la sous-commission pour la lutte contre les mesures discriminatoires et la protection des minorités qui chargea, en 1971, Martinez Cobo de réaliser une Étude du problème de discrimination à l'encontre des populations autochtones. Son rapport, publié en 1986, est l'un des premiers documents onusiens à souligner ce lien spirituel des autochtones avec la terre. Il stipule qu' «Il est essentiel de connaître et de comprendre la relation particulière, profondément spirituelle, que les populations autochtones ont avec la terre, élément fondamental de leur existence et substrat de toutes leurs croyances $[\ldots] »$.

Département d'Anthropologie, Université Lumière Lyon 2, 5 Avenue Pierre-Mendès-France 69676 Bron cedex, France. morin@univ-tlse2.fr

1 Tenu les 21-22 octobre 2004, à Montréal (Canada). Voir dans ce numéro l'article de B. Saladin d'Anglure. 


\section{La Convention 169 de l'OIT comme protection des valeurs spirituelles autochtones attachées à la terre}

Puis l'Organisation Internationale du Travail (OIT) entreprit en 1987 une révision de la Convention $\mathrm{n}^{\circ} 107$ «concernant la protection et l'intégration des populations aborigènes et autres populations tribales ou semi-tribales dans les pays indépendants» dont les visées assimilationnistes et le ton paternaliste étaient fortement critiqués par les autochtones. Le nouveau texte, connu sous le nom «Convention ${ }^{\circ} 169$ », fut adopté en 1989. Il incorpore certains droits collectifs, en particulier des droits sur les terres occupées traditionnellement par les peuples indigènes. La dimension spirituelle du territoire est là aussi prise en compte, en particulier l'article 13: «Les gouvernements doivent respecter l'importance spéciale que revêt pour la culture et les valeurs spirituelles des peuples intéressés, la relation qu'ils entretiennent avec les terres ou territoires, [...] qu'ils occupent ou utilisent d'une autre manière, et en particulier des aspects collectifs de cette relation».

\section{Un premier projet de déclaration incluant la protection des noms de lieux autochtones et des sites sacrés}

Entre temps la sous-commission avait créé en 1982 un Groupe de Travail sur les Populations Autochtones, où les experts onusiens, les délégués autochtones et les représentants gouvernementaux ont longuement discuté cette question. Ensemble, ils ont élaboré un projet de Déclaration sur les droits des Peuples Autochtones ${ }^{2}$ qui traite dans 4 articles (sur 45) des lieux sacrés et des toponymes religieux :

D'abord l'article 13: «Les Peuples Autochtones ont le droit [...] d'entretenir et de protéger leurs sites religieux et culturels [...].

Les États doivent, en collaboration avec les Peuples Autochtones concernés, prendre les mesures qui s'imposent pour faire en sorte que les lieux sacrés pour les autochtones, y compris les lieux de sépulture, soient préservés, respectés et protégés».

Puis 1'article 14: «Les Peuples Autochtones [...] ont le droit de choisir ou de conserver leurs propres dénominations pour les communautés, les lieux et les personnes».

L'article 25 stipule que «les Peuples Autochtones ont le droit de conserver et de renforcer les liens particuliers, spirituels et matériels, qui les unissent à leurs terres, à leurs territoires, à leurs eaux fluviales et côtières, et aux autres ressources qu'ils possèdent ou qu'ils occupent ou exploitent traditionnellement, et d'assumer leurs responsabilités en la matière à l'égard des générations futures». 
Et enfin l'article 29 précise que «les Peuples Autochtones ont droit à ce que la pleine propriété de leurs biens culturels et intellectuels leur soit reconnue ainsi que le droit d'en assurer le contrôle et la protection [...]».

Ce projet fut adopté en 1994 par les experts de la sous-commission de la lutte contre les mesures discriminatoires et de la protection des minorités et transmis à la Commission des Droits de l'Homme.

\section{Le second projet de Déclaration sur les droits des Peuples Autochtones bloqué par les États}

Composée de 53 États membres, la Commission des Droits de l'Homme est un organe plus politique qui évalua le texte de la sous-commission comme un simple document de travail. Elle décida alors d'élaborer elle-même un projet en créant un Groupe de Travail intersessionnel où se retrouvent chaque année depuis dix ans dans les locaux de l'ONU à Genève les représentants des États et les délégués autochtones. Une très grande majorité des autochtones souhaite s'en tenir au premier projet de la sous-commission qui promeut leurs droits collectifs alors que les États veulent l'amender ou même proposent des formulations nouvelles soulignant la dimension individuelle des droits de l'homme.

Les articles 13 et 14 font partie des droits culturels et religieux. Leur rédaction initiale ne fait pas l'objet de critiques importantes de la part des représentants étatiques. Par contre les articles 25 et 29 posent problème parce qu'ils font partie des droits fonciers que les Peuples Autochtones revendiquent et que les représentants de plusieurs États mettent en question.

En septembre 2004, lors de la dixième session du Groupe de Travail sur le projet de déclaration, les gouvernements scandinaves, la Nouvelle-Zélande et la Suisse ont proposé une série d'amendements ${ }^{3}$ pour essayer de faire avancer le processus:

Pour l'article 25, ils proposaient de supprimer les termes qui précisent la possession, l'occupation ou l'exploitation traditionnelles des terres, ou territoires par les autochtones. Les gouvernements latino-américains ont rejeté cet amendement, d'une part parce que quatorze d'entre eux avaient ratifié la convention 169 de l'OIT dont plusieurs articles reconnaissent les droits de propriété et de possession sur les terres que les autochtones occupent traditionnellement; d'autre part, parce que leur législation nationale tenait compte, dans bien des cas, de cette particularité des terres autochtones. Parmi les États favorables à l'amendement, citons le Canada. Son représentant déclara qu'il comprenait cette relation spirituelle des autochtones avec la terre mais qu'il avait quelques réserves sur la notion de propriété traditionnelle des terres et territoires. Quant aux organisations autochtones, elles ont toutes rejeté cet amendement. En particulier, la Conférence Inuit Circumpolaire, représentée par Dalee Sambo

3 Document des Nations Unies. Référence: E/CN.4/2004/WG.15/CRP.1 Information fournie par les États. 
Dorough, a montré l'importance de l'article 25 qui vise à appréhender la relation profonde que les Peuples Autochtones entretiennent avec les terres et leurs ressources et qui reflète bien leur vision du monde.

Pour l'article 29, les gouvernements scandinaves, la Nouvelle-Zélande et la Suisse ont proposé une version complètement nouvelle qui vise à supprimer la notion de pleine propriété des biens culturels et intellectuels par les autochtones ainsi que le droit d'en assurer le contrôle et la protection. Cette nouvelle version stipule simplement qu' ils «ont le droit de conserver, protéger et développer leurs biens culturels et intellectuels et les expressions tangibles de leurs biens culturels et intellectuels». Cette proposition fut discutée lors de la $3^{\text {ème }}$ semaine du Groupe de Travail sur la déclaration fin novembre 2004. Son président, Luis Enrique Chavez avait en effet obtenu une prolongation exceptionnelle de la dixième session pour tenter d'obtenir un consensus sur l'ensemble de la déclaration avant la fin du mandat du Groupe de Travail. Les autochtones étaient dans l'ensemble hostiles à cette nouvelle formulation de l'article 29 et plusieurs d'entre eux soutenaient une autre version présentée par le Conseil Saami et la Fondation Tebtebba:

«Les Peuples Autochtones ont droit à ce que la pleine propriété de leurs ressources génétiques, savoir traditionnel, expressions culturelles et patrimoine culturel leur soit pleinement reconnue, ainsi que le droit d'en assurer le contrôle et la protection.

Ils ont droit à des mesures spéciales destinées à leur permettre de contrôler, de développer et de protéger leurs sciences, leurs techniques et les manifestations de leur culture, y compris leurs ressources humaines et autres ressources génétiques, leurs semences, leur pharmacopée, leur connaissance des propriétés de la faune et de la flore, leurs traditions orales, leur littérature, leurs dessins et modèles, leurs arts visuels et leurs arts du spectacle.»

$\mathrm{Si}$ quelques représentations étatiques, en particulier latino-américaines, exprimaient leur soutien à cette version autochtone très détaillée, la grande majorité était plutôt favorable à la version «scandinave» très édulcorée citée plus haut. Comme pour l'ensemble des autres articles, un consensus n'a donc pu être trouvé et le mandat de ce Groupe de Travail se termine sur un échec puisque seulement 2 articles sur 45 ont été jusque-là adoptés.

\section{Le rapport d'Erica Daes sur la protection du patrimoine culturel autochtone}

En 1995, à la demande de la Sous-Commission de la Promotion et de la Protection des droits de l'Homme, Erica-Irene Daes a présenté un rapport ${ }^{4}$ qui comprenait des principes et des directives pour la «protection du patrimoine des populations autochtones». Elle recommandait qu'une réunion technique de l'organisation des

4 Document des Nations Unies. Référence: E/CN.4/Sub.2/1995/26 Protection du patrimoine des populations autochtones. Rapport final du Rapporteur spécial, Mme Erica-Irene Daes, présenté conformément à la résolution 1993/44 de la Sous-Commssion et à la décision 1994/105 de la Commission des Droits de l'Homme. 
Nations Unies soit organisée pour examiner les modalités de la coopération future entre les organes et institutions de l'ONU concernées par la protection du patrimoine des Peuples Autochtones.

Il faudra attendre cinq ans pour que la Commission des droits de l'Homme décide de la tenue d'un séminaire pour discuter des principes et directives de ce rapport. Il eut lieu à Genève en 2000 et réunissait 45 représentants (de gouvernements, de Peuples Autochtones, d'institutions spécialisées comme l'UNESCO, de l'OIT et de l'OMPI et d'ONG) qui apportèrent quelques modifications au texte d'E. Daes. Parmi les principes énoncés, je retiendrai que:

1) «Pour être effective, la protection du patrimoine des Peuples Autochtones devrait être largement fondée sur le principe de l'autodétermination qui comporte le droit des Peuples Autochtones de conserver et de développer leurs propres cultures et systèmes de connaissances ainsi que leurs propres formes d'organisation sociale».

2) «[...] L'utilisation et l'enseignement des connaissances des Peuples Autochtones [...] sont inextricablement liés aux terres et territoires traditionnels de chaque peuple. La maîtrise des territoires et ressources traditionnels est essentielle à la transmission ininterrompue du patrimoine des Peuples Autochtones aux générations futures, ainsi qu'à sa pleine protection».

Dans les directives, il figure une définition du patrimoine des Peuples Autochtones «(qui) revêt un caractère collectif et se compose de tous les objets, sites et connaissances, [...] dont la nature ou l'utilisation a été transmise de génération en génération et qui sont considérés comme appartenant à un peuple particulier ou à son territoire d'utilisation normale traditionnelle».

Et il est précisé plus loin (en s'inspirant des conventions de l'UNESCO) que le patrimoine des Peuples Autochtones comprend les biens culturels meubles et les biens culturels immeubles parmi lesquels «les sites sacrés qui revêtent une importance culturelle $[\ldots] »$.

\section{Autres tentatives pour protéger ce patrimoine}

Depuis 2000, deux instances onusiennes se sont plus particulièrement penchées sur cette question du patrimoine autochtone.

1) D'abord l'UNESCO, dont la déclaration universelle sur la diversité culturelle adoptée en 2001 préconise que les gouvernements élaborent des politiques et des stratégies de préservation et de mise en valeur du patrimoine culturel et naturel, notamment le patrimoine culturel oral et immatériel des Peuples Autochtones.

2) Ensuite l'OMPI (Organisation Mondiale de la Propriété Intellectuelle) qui, depuis 1998, s'intéresse à la protection du patrimoine des Peuples Autochtones. En 
2000, elle a créé un Comité intergouvernemental qui s'est engagé très activement dans le domaine des savoirs traditionnels et des expressions culturelles traditionnelles et a cherché à élargir le système de la propriété intellectuelle pour l'adapter aux communautés autochtones.

Quatre ans après son établissement, seules quelques organisations autochtones ont été accréditées, en tant qu'observateurs, auprès de ce Comité Intergouvernemental, parmi lesquelles la Conférence Inuit Circumpolaire et le Conseil Saami. Mais actuellement ce rôle d'observateur est très limité, car il ne permet pas de soumettre formellement des propositions, amendements et motions. Lors de la $5^{\text {ème }}$ session de ce Comité, les représentants autochtones ont souligné avec force la pertinence limitée des droits de propriété intellectuelle pour la protection des Savoirs Traditionnels et qu'en conséquence, tout travail à venir devrait :

1) Impliquer d'autres organisations intergouvernementales ayant des mandats plus pertinents.

2) Faire participer d'autres organisations autochtones à ce forum et tenir compte de leur point de vue.

3) Considérer le droit coutumier plutôt que la propriété intellectuelle comme point de départ.

\section{La nécessité d'un nouvel instrument juridique}

Enfin, en juillet 2004, lors de la $22^{\text {ème }}$ session du Groupe de Travail sur les Populations Autochtones à Genève, M.Yozo Yokota (un des 5 experts du GTPA) et le Conseil Saami ont présenté un document de travail ${ }^{5}$ faisant le point sur toutes ces activités internationales récentes en faveur de la protection des patrimoines traditionnels. Ils ont notamment souligné que tous ces instruments et processus internationaux ne suffisent pas à protéger le patrimoine autochtone «dont l'utilisation abusive, la représentation fallacieuse, la perte ou la destruction se poursuivent jour après jour». Il est donc important qu'un organisme des Nations Unies se voit attribuer le mandat de traiter, de façon globale, la protection de ce patrimoine.

Les auteurs ont fait remarquer que la protection du patrimoine culturel était étroitement liée à la protection des droits des Peuples Autochtones, en particulier les droits fonciers et culturels, ainsi qu'à leur droit à l'autodétermination: d'où la nécessité d'élaborer, pour la protection de ce patrimoine, un nouvel instrument procédant d'une approche fondée sur ces droits. Plusieurs problèmes restent cependant à résoudre: certains gouvernements demandent que soit définie l'expression «patrimoine culturel» car, s'il s'agit d'élaborer un texte juridiquement contraignant, ils veulent connaître son champ d'application. Quant aux Peuples Autochtones, ils affirment qu'en raison de son

Document des Nations Unies. Référence: E/CN.4/Sub.2/AC.4/2004/5 Activités Normatives. Indications destinées à orienter l'examen du projet de principes et directives pour la protection du patrimoine des Peuples Autochtones. Document de travail présenté par M. Yozo Yokota et le Conseil Saami. 
caractère holistique, leur patrimoine ne saurait être divisé en catégories. Autre élément distinctif, l'existence d'un droit coutumier autochtone qui a un rôle important à jouer dans la protection du patrimoine culturel de ces peuples.

Ces recommandations adressées au Groupe de Travail (dans le cadre de ses activités normatives) ont été soutenues au cours du débat par plusieurs organisations autochtones dont la Conférence Inuit Circumpolaire. Elle a souligné que ce futur instrument international pour la protection de ce patrimoine devrait tenir compte de la dimension collective des savoirs autochtones alors que les droits de propriété intellectuelle tels que conçus par l'OMPI avaient une dimension individuelle. 